Cultures \& Conflits

54 | été 2004

Approches critiques de la sécurité

\title{
Les études de sécurité : du constructivisme dominant au constructivisme critique
}

Alex Macleod

\section{(2) OpenEdition \\ 1 Journals}

\section{Édition électronique}

URL : http://journals.openedition.org/conflits/1526

DOI : $10.4000 /$ conflits.1526

ISSN : $1777-5345$

Éditeur :

CCLS - Centre d'études sur les conflits lilberté et sécurité, L'Harmattan

Édition imprimée

Date de publication : 1 juin 2004

Pagination : 13-51

ISBN : 2-7475-7301-X

ISSN : 1157-996X

Référence électronique

Alex Macleod, "Les études de sécurité : du constructivisme dominant au constructivisme critique », Cultures \& Conflits [En ligne], 54 | été 2004, mis en ligne le 08 janvier 2010, consulté le 30 mars 2021.

URL : http://journals.openedition.org/conflits/1526 ; DOI : https://doi.org/10.4000/conflits. 1526

Ce document a été généré automatiquement le 30 mars 2021.

Creative Commons License 


\title{
Les études de sécurité : du constructivisme dominant au constructivisme critique
}

\author{
Alex Macleod
}

Après presque une décennie de domination de la théorie des Relations Internationales aux Etats-Unis par les approches dites néoréaliste et institutionnaliste néolibérale (ou simplement néolibérale), l'arrivée du constructivisme vers la fin des années 1980 semblait apporter une véritable approche de rechange dans ce que l'on commençait à appeler le «troisième débat» de la théorie des Relations Internationales ${ }^{1}$. Le constructivisme gagna rapidement des adeptes parmi ceux qui n'acceptaient plus les termes étroits de ce que certains appelaient la synthèse "néo-néo ${ }^{2}$, au point où plusieurs observateurs parlaient déjà du constructivisme comme l'approche de l'avenir de la théorie des Relations Internationales ${ }^{3}$. Cependant, comme nous le verrons, après les premiers élans d'enthousiasme il fallut se rendre à l'évidence que, malgré ses promesses, la forme de constructivisme qui était en train de s'imposer, surtout aux Etats-Unis, constituait un courant très large, et, en fin de compte, assez prudent, qui pouvait s'accommoder facilement, certains diraient trop facilement, avec les approches déjà en place.

Dans le domaine des études de sécurité, ce constructivisme dominant a produit quelques études intéressantes, mais qui reflètent toutes cette prudence existentielle, autant sur le plan épistémologique ${ }^{4}$ que sur celui de l'ontologie. Et pourtant, plusieurs auteurs ont trouvé dans le constructivisme suffisamment d'éléments potentiellement novateurs pour les convaincre d'engager le dialogue à l'intérieur d'un cadre constructiviste, et de créer les bases d'une approche, encore assez hétéroclite, que nous qualifions de constructivisme critique et qui commence à proposer des pistes de réflexion intéressantes pour les études de sécurité.

Même aujourd'hui, toute remise en cause des approches traditionnelles envers la sécurité prend pour point de départ la critique du néoréalisme, avec, comme cible préférée, le livre Theory of International Politics de Kenneth Waltz, paru en 1979. La 
conception néoréaliste de la sécurité, si bien articulée par Waltz, est très simple : il faut assurer la survie de l'Etat ${ }^{5}$. La sécurité se réduit donc, chez les néoréalistes à la défense militaire et les études de sécurité se confondent avec les études stratégiques ${ }^{6}$. Même si, dans les années 1980, on commençait à questionner cette vision étroite de la sécurité ce n'est qu'après la fin de la Guerre Froide que l'on peut parler d'un renouveau de la pensée sécuritaire.

Le but principal de cet article sera de définir ce que l'on veut dire exactement par constructivisme critique et d'évaluer sa contribution aux études de sécurité. Pour ce faire, nous commencerons par nous interroger sur ce qui distingue ce type de constructivisme du courant dominant. Ensuite, nous tenterons de faire ressortir les traits des études de sécurité que proposent les constructivistes critiques. Nous aborderons cette démonstration en deux étapes. En premier lieu, nous examinerons deux tentatives importantes de concevoir autrement la sécurité, les études critiques de sécurité et le postmodernisme, avec lesquelles on confond trop facilement le constructivisme critique. La deuxième étape se concentrera plus spécifiquement sur une comparaison entre les études de sécurité proposées par le constructivisme dominant et celles qu'offre le constructivisme critique.

\section{A la recherche d'une définition du constructivisme}

Définir le constructivisme n'est pas tâche facile. Il est probablement plus simple de commencer par dire ce qu'il n'est pas. En premier lieu, il ne faut pas le confondre avec la Théorie Critique ${ }^{8}$ et le postmodernisme, même si ces deux derniers partagent avec le premier une vision d'un monde qui serait essentiellement « socialement construit ». Il est important d'établir cette distinction, car partisans et adversaires tendent, avec plus ou moins de bonne foi, à les amalgamer sous l'étiquette générale de "théorie critique ». C'est le cas, notamment, du néoréaliste John Mearsheimer' ${ }^{9}$, et de la réaliste Jennifer Sterling-Folker, qui parle du postmodernisme, «ou de ce que l'on appelle plus communément la théorie critique», comme un des prédécesseurs du constructivisme sur le plan théorique ${ }^{10}$. Comme nous le verrons, il y a des différences fondamentales entre les trois approches qui sont irréconciliables, même si, à des degrés divers, elles adoptent des positions critiques à l'égard des théories positivistes.

\section{Les deux grands courants constructivistes}

Il n'est pas aisé de faire totalement justice à toutes les approches théoriques qui se réclament $\mathrm{du}$ constructivisme ${ }^{11}$. Au risque de commettre une simplification grossière de la situation, nous pensons que l'on assiste essentiellement à l'émergence de deux grands courants constructivistes, qui comprennent bien sûr des nuances significatives, voire des divergences de vue, à l'intérieur de chacun d'entre eux. On pourrait qualifier le premier de "dominant $»^{12}$. Il s'inspire largement des travaux d'Alexander Wendt, et nous donnons au deuxième l'étiquette de "critique ». Celle-ci a une signification beaucoup plus précise que celle proposée par Ted Hopf, qui se contente de distinguer le constructivisme « dominant » et le " constructivisme critique », ou tout simplement la "théorie critique », qui « rejette l'idée qu'un fondationnalisme minimal ou contingent soit possible ou désirable» ${ }^{13}$. La distinction de Hopf revient donc à assimiler le constructivisme critique au postmodernisme, ce qui serait une grave erreur, 
malheureusement assez répandue. Comme nous le verrons, ce constructivisme critique a des caractéristiques qui lui sont propres et que partagent plusieurs auteurs, qui ne se retrouvent ni dans le constructivisme dominant, ni dans le postmodernisme

Deuxièmement, et là-dessus il y a un accord quasi-général, le constructivisme ne constitue pas encore un véritable paradigme au sens khunien du terme. On l'a décrit comme une "approche $»^{14}$, une «approche ou un style $»^{15}$, une «méthode plus que toute autre chose ${ }^{16}$, une " approche de portée théorique envers l'étude des relations internationales ${ }^{17}$, ou tout simplement comme un "ensemble d'approches interliées, plutôt qu'une approche complètement cohérente $»^{18}$.

Enfin, il faut placer le constructivisme dans le contexte plus général de la théorie des Relations Internationales. Cet exercice devient difficile, puisqu'il s'agit d'essayer de définir les lignes de division dans la discipline, telle que celle-ci a évolué depuis les vingt dernières années. Par exemple, on distingue souvent, du moins dans la littérature américaine, le "positivisme» du "postpositivisme», ou le "rationalisme» du "réflectivisme $~^{19}$. En agissant ainsi, on crée l'impression d'une division profonde et symétrique entre deux camps cohérents et bien organisés, qui ne correspond nullement à une réalité beaucoup plus complexe. Si l'on peut démontrer sans difficulté la cohérence assez forte des théories qui se réclament aujourd'hui du positivisme, il n'en va pas de même des approches dites postpositivistes. Par exemple, les partisans de la Théorie Critique mettent en avant des positions fondationnalistes ${ }^{20}$ que tous les postmodernistes rejettent du revers de la main.

Il existe néanmoins une ligne de démarcation assez nette entre les divers types de théorie. D'un côté on retrouve ceux qui mettent l'accent sur la nécessité d'adopter une approche «scientifique " pour l'étude d'un monde essentiellement matériel, fondée sur une épistémologie empiriste, sur la séparation entre faits et valeurs, et sur la recherche de régularités dans le comportement d'acteurs (Etats) autonomes. Et de l'autre, on rencontre ceux qui font preuve de scepticisme à l'égard de l'« objectivité » à laquelle aspirent les premiers, et qui considèrent qu'il importe de savoir non seulement pourquoi des acteurs se comportent d'une certaine façon mais aussi comment ils en sont arrivés là. Pour rendre compte de cette distinction, Steve Smith préfère parler de la différence entre théories "explicatives» et théories "constitutives" ou « interprétatives », qu'il définit de la façon suivante : « une théorie explicative voit le monde comme quelque chose d'extérieur à nos théories à son sujet; par contre, une théorie constitutive croit que nos théories participent à la construction du monde $»^{21}$. Aborder les approches de cette façon présente plusieurs avantages. Premièrement, elle ne postule pas une cohérence artificielle, ni d'un côté ni de l'autre. Ensuite, cela évite l'idée, implicite dans les deux premières classifications, que l'on appartient forcément entièrement à l'un ou l'autre camp. Enfin, cela permet une plus grande souplesse dans la caractérisation des diverses approches. Mais avant d'essayer de catégoriser le constructivisme selon le schéma proposé par Smith, nous devons tenter d'identifier les traits principaux de cette approche.

A la base, pour reprendre les termes de celui qui a lancé cette approche en théorie des Relations Internationales, le constructivisme " prétend que les gens font la société, et la société fait les gens $»^{22}$. Plus spécifiquement en Relations Internationales, cette idée se fonde sur quelques prémisses, qu'Alexander Wendt résume de la façon suivante : « (1) que les structures de l'association humaine sont déterminées principalement par des idées partagées plutôt que par des forces matérielles, et (2) que les identités et les 
intérêts d'acteurs réfléchis sont construits par ces idées plutôt que donnés par la nature $»^{23}$.

Selon cette vision des relations internationales, les acteurs ou les agents ont des identités qui participent à la création de leurs intérêts, qui, eux, influent sur leur comportement et leur relation avec d'autres acteurs ou d'autres agents. Bien que Wendt mette l'accent sur les "idées partagées » et sur l'« intersubjectivité », les constructivistes ne prétendent pas que les facteurs matériels soient sans importance, mais, contrairement aux néoréalistes, ils croient que les idées comptent et que les identités, et donc les intérêts, ne peuvent être traités comme de simples données immuables. Les relations entre acteurs internationaux, c'est-à-dire les Etats, ne seraient pas régies uniquement par la recherche de la sécurité, mais comprendraient aussi la création, le respect et la mise en vigueur de normes, qui sont souvent intériorisées pour faire partie de l'identité des acteurs.

$\mathrm{Au}$ cœur du projet constructiviste se trouve la question des rapports entre agence et structure. Selon Smith, «dans le débat entre agence et structure, on se demande si les explications du comportement social humain doivent être fondées sur les intentions des acteurs et leur définition de la situation, ou sur un modèle des sciences naturelles, dans lequel on n'aurait besoin d'aucune agence consciente $»^{24}$. En fait, si la plupart des constructivistes, du moins chez les partisans du constructivisme dominant, prétendent que l'agence et la structure se constituent réciproquement, qu'elles se «coconstituent ", ils penchent tout de même du côté de la structure, au point où Wendt décrit le constructivisme comme une sorte d' "idéalisme structurel » ${ }^{25}$ et avoue que son objectif n'est pas d'« expliquer les identités et les intérêts des Etats ${ }^{26}$.

Partisans et adversaires du constructivisme s'entendent sur ses apports nouveaux sur le plan de l'ontologie et sa prudence sur celui de l'épistémologie. Sur ce dernier point, il existe un consensus général selon lequel le constructivisme dominant ne s'est pas trop éloigné des approches positivistes ${ }^{27}$. Et malgré ses prétentions à renouveler l'ontologie des Relations Internationales, le constructivisme dominant reste très traditionnel sur un point fondamental. Il met toujours l'Etat au centre de ses analyses, même s'il refuse le modèle de l'Etat « unitaire et rationnel » du néoréalisme et du néolibéralisme. On ne doit pas s'étonner de cette position, compte tenu de l'importance que cette forme de constructivisme accorde aux concepts d'identité nationale et d'intérêt national. Cette préoccupation vis-à-vis du «national » et du rôle de l'Etat a eu un effet décisif sur les approches constructivistes dans le domaine de la sécurité. Il semble donc tout à fait normal que le texte constructiviste sur la sécurité le plus cité porte le titre de The Culture of National Security ${ }^{28}$.

Les adeptes du constructivisme dominant se servent de leur proximité avec le positivisme sur le plan épistémologique et de leurs innovations ontologiques pour proclamer qu'ils peuvent faire le pont entre positivisme et postpositivisme. Wendt a déclaré son espoir de trouver une "voie moyenne fondée sur des principes philosophiques» $»^{29}$, tandis qu'Emanuel Adler parle de $"$ saisir le milieu du terrain $»^{30}$. Sans aller aussi loin, Martha Finnemore et Kathryn Sikkink ont conclu que l'opposition entre rationalistes et constructivistes sur les questions clés de normes et d'identités constitue une ligne de faille qui ne peut être défendue «ni sur le plan empirique ni sur le plan théorique $»^{31}$. Bill McSweeney a ironisé sur ce « consensus grandissant parmi les constructivistes " selon lequel ils sont lancés dans une "entreprise irénique capable d'apporter l'harmonie intellectuelle à une discipline menacée par les critiques 
dissidents de son courant majoritaire positiviste $»^{32}$. Pour sa part, Steve Smith applaudit l'effort, mais condamne sa futilité parce que Wendt, et donc les tenants du constructivisme dominant en général, ont défini le constructivisme «d'une manière étroite, qui est acceptable pour les rationalistes, mais qui ne serait pas acceptée par les réflectivistes ${ }^{33}$. Cette évaluation a été confirmée par trois auteurs, un constructiviste (du courant dominant), un néolibéral et un réaliste, qui ont plaidé en faveur d'une convergence entre le constructivisme et les approches positivistes :

Les deux perspectives analytiques se concentrent d'une façon ou d'une autre sur la connaissance commune - le constructivisme sur la manière dont elle est créée, le rationalisme [c'est-à-dire le positivisme, nda] sur la manière dont elle affecte la prise de décisions stratégiques. Au cœur du projet constructiviste on trouve l'explication des variations dans les préférences, les stratégies disponibles, et la nature des acteurs à travers l'espace et le temps. Au cœur du projet rationaliste on trouve l'explication des stratégies, des préférences données, l'information et la connaissance commune. Aucun des deux projets ne peut être complet sans l'autre ${ }^{34}$.

Il est évident qu'en tentant d'établir une sorte d'évolution naturelle entre le constructivisme et les deux approches positivistes qui ont dominé la théorie des Relations Internationales aux Etats-Unis depuis les années 1980, les tenants du constructivisme dominant sont en train de préparer le terrain pour une OPA en douceur sur l'avenir de la théorie des Relations Internationales. L'épistémologie resterait intacte, et l'élargissement vers l'ontologie ne bouleverserait pas trop la tradition stato-centriste. En ce sens Ole Wæver a eu sans doute raison de parler du "potentiel presque ahurissant» du constructivisme dominant de devenir une "métathéorie qui comprendrait toutes les autres» ${ }^{35}$.

Le constructivisme critique n'aspire nullement à occuper la position du nouveau paradigme de la théorie des Relations Internationales. Ses ambitions sont beaucoup plus modestes. Comme toute approche critique, il cherche avant tout à provoquer la réflexion et le dialogue et d'éviter la sclérose qui guette toute pensée dominante. Par contre, il veut rester dans la famille constructiviste parce qu'il partage sa vision générale d'un monde socialement construit, tout en reprochant au courant constructiviste dominant son conservatisme épistémologique et ontologique. Il veut y rester aussi parce que, comme le dit très candidement George Marcus, les constructivistes critiques «ne veulent manifestement pas être ignorés par la majorité dans la même mesure » que les critiques postmodernes, avec qui, par ailleurs ils sont «fortement alliés sur le plan intellectuel $»^{36}$. Karin Fierke exprime cette position d'une façon un peu différente quand elle place le constructivisme critique à la frontière entre l'empirique et le critique, c'est-à-dire entre le constructivisme dominant et le postmodernisme ${ }^{37}$. Si on ne peut parler d'un véritable projet constructiviste critique, la plupart des sympathisants du constructivisme critique souscriraient sans difficulté aux trois principes proposés par Weldes et al. :

«Ce que l'on comprend comme réalité est socialement construit.

Les constructions de la réalité reflètent, décrètent, et réifient des relations de pouvoir. À leur tour, certains agents ou groupes d'agents jouent un rôle privilégié dans la production et la reproduction de ces réalités.

Une approche constructiviste critique dénaturalise les constructions dominantes, offre des indications pour la transformation du sens commun, et facilite l'imagination de modes de vivre alternatifs. Elle problématise aussi les conditions de ce qu'elle affirme ; en d'autres termes, un constructivisme critique est aussi réflexif $»^{38}$. 
Le réflexivisme est un aspect important de toute théorie qui se veut critique. Il est fondamental pour le constructivisme critique. Il signifie avant tout que le chercheur doit être conscient à la fois des prémisses de sa propre pensée, voire de ses préjugés, et des valeurs et des normes qui sous-tendent toute théorie. Il ne peut y avoir séparation entre valeurs et faits, ou entre théorie et pratique. Toute analyse doit toujours tenir compte du contexte social. Et si le réflexivisme souscrit à l'idée de l'incommensurabilité entre paradigmes ou courants théoriques qui s'opposent sur le plan épistémologique, il croit que le dialogue est possible, voire souhaitable entre eux ${ }^{39}$.

Enfin, les constructivistes critiques se distinguent des partisans de la Théorie Critique par le fait qu'ils ne se réfèrent ni à Gramsci ni à l'Ecole de Francfort, et rarement au marxisme, et ne partagent pas leur objectif central d'émancipation. A la différence de ces derniers, ils sont souvent moins réticents à se référer à certains concepts postmodernes, et à des penseurs comme Michel Foucault et Jacques Derrida, mais acceptent explicitement ou implicitement une forme de fondationnalisme. Ils font ainsi preuve d'une ouverture au dialogue avec ces derniers qui semble impensable pour les partisans du constructivisme dominant ${ }^{40}$. Ils sont donc beaucoup mieux placés que les tenants du constructivisme dominant pour occuper non pas une "voie moyenne" illusoire mais la meilleure place pour maintenir le débat entre postpositivistes et positivistes.

\section{Etudes critiques de sécurité et postmodernisme}

Comme nous l'avons vu, il existe beaucoup de confusion autour de ce que l'on entend par "postpositivisme", surtout quand il s'agit de distinguer la Théorie Critique du postmodernisme. Il est vrai que ces deux approches adoptent des positions très critiques envers les théories dominantes en Relations Internationales, mais les différences entre elles sont relativement nettes, pour ceux qui veulent les voir. La différence la plus évidente réside dans leurs objectifs. Les partisans de la Théorie Critique ne se contentent pas d'attaquer le statu quo intellectuel et politique et le positivisme. Ils proposent aussi des alternatives radicales et ne cachent nullement leur fondationnalisme. En revanche, les postmodernistes rejettent toute position qui se voudrait quelque peu fondationnaliste. Ainsi, dans sa discussion du concept de la sécurité, James Der Derian insiste sur le fait qu'il n'est pas "à la recherche d'une 'sécurité alternative' ${ }^{41}$. Comme nous le verrons, cela ne les empêche pas de proposer d'autres façons de conceptualiser la sécurité.

\section{Les études critiques de sécurité}

Selon Bill McSweeney, les «études critiques de sécurité » représentent la «plus clairement focalisée » des approches postpositivistes à l'égard de la sécuritét2 ${ }^{42}$ tandis que Steve Smith les a louées comme «la critique la plus soutenue et la plus cohérente des études traditionnelles de sécurité $»^{43}$. Il nous semble, cependant, que les réalisations des études critiques de sécurité jusqu'ici nous invitent à un peu plus de prudence. Les études critiques de sécurité, nées en $1994^{44}$ proviennent en grande partie des réflexions des tenants de la Théorie Critique, bien qu'elles soient plus hétéroclites. Là encore on peut distinguer l'approche assez large des Canadiens Keith Krause et Michael Williams, qui met l'accent sur la critique des conceptions traditionnelles de la sécurité, et celle de 
Ken Booth et de Richard Wyn Jones, où le projet émancipateur est beaucoup plus présent. La recherche d'une définition plus précise des études critiques de sécurité n'est pas facilitée par le fait que le titre de Théorie Critique soit attribué à des approches qui s'inspirent de deux courants marxisants assez différents, celui du néogramscisme et celui de l'Ecole de Francfort ${ }^{45}$. Elles partagent, cependant, ce que Nicholas Rengger appelle le "projet émancipateur »" et qui est devenu la caractéristique commune de la Théorie Critique en Relations Internationales.

Krause et Williams commencent leur étude de la sécurité avec la célèbre distinction que propose le néogramscien Robert Cox entre deux types de théorie, celle qui a pour objet principal la résolution de problèmes (problem-solving theory) et qui se satisfait du statu quo politique et social, et la Théorie Critique, qui porte un projet de changement. Mais ils insistent sur le fait que leur conception de la « critique » est " censée impliquer plus une orientation envers la discipline qu'une étiquette théorique précise $\star^{47}$. La variété des approches utilisées par les contributeurs à leur ouvrage, qui comprennent postmodernisme, constructivisme, Théorie Critique, et même une " perspective réaliste subalterne ", confirme largement cette vision de la notion de critique. L'objectif principal recherché n'est donc pas de fonder une école de pensée, mais plutôt d'ouvrir le débat sur ce que signifie la sécurité et sur les possibilités d'y penser autrement.

Cette approche plutôt éclectique envers la critique n'empêche pas Krause et Williams de proposer des pistes à explorer. En premier lieu, ils favorisent « un déplacement vers des modèles de compréhension plus interprétatifs [au sens large] $»^{48}$, ce qui les place dans le camp des partisans des théories constitutives. Cela les amène à proposer une critique sans appel du néoréalisme et de sa conception étroite de la sécurité. Ce faisant, cependant, ils se limitent à indiquer les difficultés que l'on rencontre en tentant de proposer des alternatives, surtout sur le plan ontologique. Ainsi ils rejettent un simple élargissement du champ de la sécurité, puisque la «simple articulation d'une large gamme de menaces à la survie ou au bien-être humain nouvellement émergentes ou nouvellement reconnues ne fera pas bouger les études de sécurité de leurs préoccupations traditionnelles» ${ }^{49}$. Ils prônent plutôt une reconceptualisation de la sécurité, ce qui veut dire qu'il faut répondre directement à une question centrale pour la Théorie Critique : «Existe-t-il un fondement pour l'ordre politique qui peut fournir un référent et un acteur dans un monde en voie de mondialisation $»^{50}$ ? Autrement dit, les études critiques de sécurité ne peuvent se contenter d'être purement négatives et se réfugier dans la déconstruction. Elles doivent aussi adopter un "programme reconstructif $»^{51}$.

Bien que Krause et Williams acceptent la définition de Cox de la Théorie Critique, ils ne font que peu de référence à la notion d'émancipation. Par contre, Ken Booth et Richard Wyn Jones, tous les deux adeptes de l'Ecole de Francfort, la mettent au cœur de leur vision des études critiques de sécurité. Pour Booth, l'idée de l'émancipation signifie «libérer les gens, en tant qu'individus et groupes, des contraintes sociales, physiques, économiques, politiques et autres, qui les empêchent d'entreprendre ce qu'ils choisiraient de faire librement ", tout en reconnaissant qu'au fur et à mesure que "les circonstances changeront, les objectifs de l'émancipation changeront aussi $»^{52}$. Les tenants de cette version des études critiques proposent une conceptualisation de la sécurité qui comprend les trois processus d'approfondissement, d'élargissement et d'extension. Selon Wyn Jones, l'approfondissement suppose une critique épistémologique et ontologique des études de sécurité traditionnelles et une « tentative 
plus profonde de réfléchir sur ce que l'émancipation pourrait signifier en termes d'institutions et de pratiques alternatives ${ }^{53}$. Comme Krause et Williams, Wyn Jones se méfie de l'idée d'élargir le concept de sécurité simplement en le séparant en cinq domaines distincts, comme le font Barry Buzan et les autres membres de l'Ecole de Copenhague. Il s'agit avant tout de reconnaître que la définition de la sécurité est toujours en train de se transformer et de s'élargir. On ne peut l'enfermer donc dans un carcan défini à l'avance. Enfin, en proposant l'extension du concept de sécurité, Wyn Jones et Booth visent surtout ce qu'ils appellent l'étatisme (statism) de l'approche traditionnelle, vision qui, non seulement, privilégie l'Etat comme acteur international, mais qui signifie aussi une "prétention normative» selon laquelle "en termes politiques, on devrait accorder aux Etats une valeur élevée, sinon la plus élevée, en soi $\aleph^{54}$. Pour les partisans des études critiques, on ne doit pas limiter les entités qui peuvent devenir un objet référent de la sécurité, et donc il n'est pas question de privilégier d'office une entité par rapport à une autre, bien qu'ils aient un penchant particulier pour l'individu comme « ultime référent $»^{55}$.

A ce stade de leur évolution, les études critiques de sécurité tendent, avant tout, à soulever des questions pertinentes et à suggérer dans quelle direction on doit chercher les réponses. Il est vrai, bien sûr, que la notion d'« émancipation » semble constituer une option normative indiscutable, mais en la proposant, les partisans des études critiques tiennent pour acquis qu'il existe déjà une entente sur ce qu'elle veut dire exactement et un consensus sur l'idée qu'elle devrait constituer le point central des études de sécurité. Manifestement, c'est loin d'être le cas. Les études critiques de sécurité proposent l'ébauche d'un programme prometteur, mais représentent pour le moment plus un projet en chantier qu'une véritable approche théorique ${ }^{56}$.Les approches postmodernes des études de sécurité

Malgré ses positions postpositivistes, la Théorie Critique s'insère assez facilement dans le débat général sur la théorie des Relations Internationales aux Etats-Unis. En revanche, le postmodernisme et le poststructuralisme ont certes secoué le cocotier, mais tendent à être traités avec dédain par les partisans des approches positivistes. Dans leur survol de l'histoire de la théorie des Relations Internationales, Katzenstein, Keohane et Krasner écartent la contribution du postmodernisme en prétendant que celui-ci «tombe nettement en dehors de l'entreprise des sciences sociales, et risque dans les recherches en relations internationales de devenir auto-référentiel et désengagé du monde $\aleph^{57}$. Nous tenterons de démontrer que de telles déclarations sousestiment l'importance de la contribution du postmodernisme au débat sur la sécurité.

Encore plus que le constructivisme, le postmodernisme échappe aux définitions simples et indiscutables ${ }^{58}$. Même la distinction entre postmodernisme et poststructuralisme pose problème. John Vasquez prétend que c'est la question du relativisme qui marque la différence la plus importante entre les deux, les poststructuralistes ayant tendance à jouer avec cette idée, tandis que les postmodernistes l'assument totalement ${ }^{59}$. Pauline Rosenau voit aussi, essentiellement, plus une différence d'accent que de substance, les postmodernistes étant "plus orientés vers la critique » et les poststructuralistes mettant l'accent sur « la méthode et des questions épistémologiques », mais ajoute que "les différences semblent peu porter à conséquences $»^{60}$. Pour simplifier les choses, nous parlerons donc uniquement de postmodernisme et de postmodernistes.

Rosenau a résumé très succinctement ce que le postmodernisme cherche à accomplir : il « conteste toute vision du monde qui se veut globale ou prétend tout comprendre ", 
et rejette «les méta-récits logocentriques, transcendantaux et totalisants qui anticipent toutes les questions et fournissent toutes les réponses ». Donc il n'a pas pour objectif de "formuler un ensemble alternatif de postulats, mais d'enregistrer l'impossibilité d'établir tout fondement aux connaissances $»^{61}$. Le postmodernisme exprime le scepticisme et la méfiance à l'égard de toute tentative de construire des théories générales. Il est donc résolument anti-fondationnaliste.

Il faut situer l'approche postmoderne à l'égard de la sécurité dans le contexte plus large de sa conceptualisation des Relations Internationales. Celle-ci part du rejet de toute version positiviste des sciences sociales, et surtout des fondements épistémologiques et ontologiques du néoréalisme ${ }^{62}$. En pratique, cela veut dire que les postmodernistes refusent toute division rigide entre le monde intérieur de la vie politique nationale et le monde extérieur des "relations internationales", surtout sous sa forme de relations inter-étatiques. Bradley Klein définit donc la tâche des sciences sociales comme étant celle de donner une "voix et de la clarté aux multiples forces et mouvements sociaux qui participent à la constitution de la vie internationale $»^{63}$. Une des cibles principales de cette attaque contre la théorie traditionnelle des Relations Internationales est l'Etat et le concept de souveraineté qui l'accompagne. La question de l'Etat et de la souveraineté dans la littérature postmoderne ne concerne pas tant la recherche d'autres référents ou acteurs internationaux que la tendance des néoréalistes et des néolibéraux à les présenter comme non problématiques et hors contexte. Selon R.B.J. Walker:

«Les prétentions souveraines des Etats n'expriment pas un simple fait de la vie, comme ceux qui travaillent avec les conceptions dominantes de la sécurité le répètent si souvent. On ne peut non plus faire comme si elles n'existaient pas, au nom d'une quelconque humanité commune, comme nombre de ceux qui défendent une version alternative de la sécurité semblent souvent le croire. Elles sont une articulation spécifique de relations d'universalité/particularité et de soi/Autre, une articulation qui dépend, en fin de compte, de la capacité de distinguer une frontière territoriale ou spatiale entre une politique historique à l'intérieur et une nonpolitique simplement contingente à l'extérieur de l'Etat moderne $"^{64}$.

Se concentrer uniquement sur l'Etat déforme aussi la conceptualisation de la sécurité. Ce thème, développé en particulier par David Campbell, se trouve au cœur d'une des principales questions soulevées par les penseurs postmodernes en Relations Internationales. Dans son interprétation de la politique étrangère et de sécurité des Etats-Unis pendant la Guerre Froide, Campbell avance l'idée selon laquelle la politique étrangère ne touche pas seulement les relations avec d'autres Etats, mais est avant tout la «reproduction d'une identité instable au niveau de l'Etat, et la limitation des contestations de cette identité ${ }^{65}$. Ainsi, pour l'Etat, on peut comprendre l'identité comme le résultat de pratiques exclusionnistes où les éléments qui résistent à une identité stable à l'intérieur' sont liés, par un discours de 'danger', avec des menaces identifiées et situées à l'extérieur. Il en résulte que l'on construit des frontières, démarque des espaces, incorpore des normes de légitimité, privilégie des interprétations de l'histoire et marginalise les alternatives ${ }^{66}$.

Autrement dit, les Etats et les identités collectives vont ensemble et participent à un processus d'« établissement de limites d'inclusivité et d'exclusivité aux marges ou aux frontières de la souveraineté» ${ }^{67}$. Le politique qui se limite à l'espace défini par l'Etat crée ainsi les conditions de l'exclusion et du refus de la différence. 
Etant donné cette vision des affaires mondiales, les postmodernistes reconnaissent les difficultés inhérentes à toute reconceptualisation de la sécurité. Selon Walker, il s'agit de repenser "le caractère et la situation du 'politique'", ce qui "exige un degré considérable de scepticisme à l'égard des principes modernes de l'autonomie et de la subjectivité souveraine ». Il faut aussi «répondre en particulier aux questions de qui assure-t-on la sécurité, et sous quelles conditions ${ }^{68}$.

Face à ces questionnements, les postmodernistes rejettent l'idée que nous pouvons simplement créer un monde "sécurisé ». Ils nous invitent plutôt à «imaginer un nouveau dialogue de la sécurité, non pas dans la poursuite d'un objectif utopique mais dans la reconnaissance du monde tel qu'il est, autre que nous $»^{69}$. Comme le dit si bien Campbell, «nous ne pouvons éviter le danger et chercher à entrer dans une situation sans risque (...) [cela] fait partie de nos rapports avec le monde et peut être ressenti de façon positive aussi bien que négative $\aleph^{70}$. Cependant, quand il est question de définir ce que voudrait dire une telle reconceptualisation de la sécurité, les réflexions postmodernes tendent à devenir un peu plus traditionnelles, malgré un langage et une terminologie qui le sont beaucoup moins. Ainsi, Walker nous met en garde contre la tentation de rejeter "trop rapidement» les notions dominantes de la sécurité, parce que l'on a fait des progrès qui nous permettent d'abandonner les postulats sur l'inévitabilité du conflit en faveur des possibilités de coopération, que les affirmations au sujet d'alternatives « exprimeront probablement un engagement continu, bien que voilé, envers ces pratiques censément désuètes», et, enfin, que ces affirmations «peuvent être lues comme des expressions d'un engagement normatif hégémonique envers la manière dont le monde doit être $»^{71}$. Cela dit, pour les postmodernistes la "dévaluation soudaine du discours dominant [sur la sécurité] (...) nous donne une occasion unique pour comprendre le processus à travers lequel nous posons nos questions sur la stratégie et la sécurité et commençons à aller au-delà de ce processus $»^{72}$.

Il n'est donc pas étonnant que les postmodernistes partagent le scepticisme des partisans de la Théorie Critique à l'égard de l'élargissement de la notion de sécurité, non pas parce qu'ils souhaitent garder une conception étroite de celle-ci, mais plutôt parce qu'une telle opération "pose une gamme de problèmes politiques qu'il faudra thématiser $\aleph^{73}$. L'élargissement de la vision traditionnelle de la sécurité produit des effets pervers, puisque « lorsque l'on fournit diverses formes de sécurité, on reproduit aussi des insécurités, souvent par des façons qui soit sapent en fait la production initiale de sécurité soit perpétuent simplement les problèmes pour lesquels elles sont censées fournir des solutions ${ }^{74}$.

Les postmodernistes soumettent les tentatives de l'Ecole de Copenhague d'étendre le concept de sécurité à un examen tout particulier. Et cela nous donne un aperçu de la manière dont ils conçoivent la "nouvelle pensée » sécuritaire. Walker s'attaque à la façon dont Buzan traite de la sécurité nationale, qui est une " conception holiste » où celle-ci est « comprise dans un contexte plus large de 'sécurité systémique'» qui « reste presque entièrement préoccupé par la sécurité des Etats, bien qu'il s'agisse d'une sécurité des Etats comprise à l'intérieur d'un concept plus large.»Ce faisant, Buzan passe à côté de « la plupart des questions, plus difficiles, à propos de la signification possible de la sécurité dans le monde contemporain », et parmi lesquelles Walker cite " la dégradation écologique, le développement inégal à l'intérieur et entre les Etats ou la façon dont les Etats continuent à être la menace principale pour la sécurité des gens 
dans beaucoup d'endroits $»^{75}$. Campbell indique aussi que l'environnement, un des cinq secteurs du nouveau champ de sécurité proposé par l'Ecole de Copenhague, peut être reconceptualisé comme un "danger qui peut être articulé en termes de stratégies sécuritaires déterritorialisées, qui impliquent une coopération communautaire, qui reconfigurent les relations économiques (...) [et] qui peuvent servir à encadrer une interprétation différente du 'politique' ${ }^{76}$. Enfin, Roxanne Lynne Doty propose une critique nuancée des deux concepts-clés de l'Ecole de Copenhague, celui de sécurisation et celui de sécurité sociétale. Elle note que la conceptualisation de la sécurité comme « acte de langage » qu'offre Ole Wæver est utile " pour comprendre la nature construite de la sécurité", mais il est trompeur de "limiter notre compréhension de la sécurisation à un processus instrumental qui est contrôlé par des élites et ceux qui détiennent le pouvoir $\aleph^{77}$. Quant à la notion de sécurité sociétale, Doty la loue comme "un renouveau fondamental de la pensée sur la sécurité " et un "changement important dans les préoccupations traditionnelles exprimées dans les études de sécurité », mais elle "ne problématise ni la sécurité elle-même ni la logique qui la soustend", logique où il devient impossible d'imaginer un "genre de sécurité qui ne dépende pas d'une certaine compréhension du danger, et d'une compréhension particulière de soi et de l'autre ». Elle se méfie aussi de la politique identitaire, associée à l'idée de sécurité sociétale et qui « implique des tentatives de réaffirmer l'identité de ceux qui appartiennent à une société particulière et en même temps de définir ceux qui en seront exclus $»^{78}$. Comme nous le verrons, Doty rejoint, dans un vocabulaire un peu différent, les analyses des constructivistes critiques.

\section{Des études de sécurité du constructivisme dominant à celles du constructivisme critique}

La pensée constructiviste dans les études de sécurité a emprunté le même chemin que le constructivisme en théorie des Relations Internationales. D'un côté, un courant dominant s'est établi, en particulier autour du livre de Peter Katzenstein, The Culture of National Security : Norms and Identity in World Politics, et, dans une moindre mesure, celui d'Emanuel Adler et Michael Barnett, Security Communities ${ }^{79}$, publiés respectivement en 1996 et 1998. De l'autre côté, on retrouve des constructivistes critiques tels que Didier Bigo, Karin Fierke, Jef Huysmans, Bill McSweeney et Jutta Weldes, qui se démarquent du constructivisme dominant par leurs critiques des idées qui sous-tendent l'ouvrage de Katzenstein, leur insatisfaction à l'égard des travaux de l'Ecole de Copenhague, leur approche sociolinguistique, et par leur rejet du positivisme.

Comme on pourrait s'y attendre, il n'est pas facile de définir avec exactitude une approche qui rassemble des gens de sensibilités philosophiques et politiques assez distinctes. On remarquera, cependant, certaines affinités qui les unissent dans un courant qui commence à se démarquer du constructivisme dominant. Qu'ils utilisent carrément le terme " constructivisme critique », comme Weldes ou Fierke, ou qu'ils se qualifient de «constructiviste structuraliste » (Bigo), de «social-constructiviste» (Huysmans), ou même de "constructionniste social » (McSweeney), ils se réclament tous sans ambages du constructivisme, mais rejettent toute appartenance au constructivisme dominant. 


\section{Le constructivisme dominant face à la sécurité}

La définition du constructivisme proposé par Adler et Barnett, exprime assez bien l'orientation générale de la position majoritaire : «il prétend que les acteurs internationaux sont enchâssés dans une structure qui est à la fois normative et matérielle [c'est-à-dire qu'elle contient des règles et des ressources] et il tient compte de la possibilité que, sous les conditions appropriées, les acteurs peuvent générer des identités et des normes qui sont liées à une paix stable $»^{80}$. Il n'en faut pas plus pour que McSweeney qualifie une telle approche de "constructivisme néolibéral», "qui a beaucoup plus en commun avec l'accent que les libéraux-rationnalistes mettent sur la coopération transnationale, les institutions et les normes, et sur la primauté non problématique de l'Etat qu'avec un programme de recherche fondé sur les principes réflexivistes de la continuité du collectif et de la co-constitution de l'agence et de la structure $»^{81}$.

La présentation que fait McSweeney de l'épistémologie et de l'ontologie du constructivisme dominant est bien fondée. Katzenstein poursuit dans son livre l'objectif d'« élargir la position moyenne pour une analyse de la sécurité nationale ${ }^{82}$, tandis qu'Adler et Barnett prétendent que les théories des Relations Internationales " peuvent et doivent occuper une position moyenne pragmatique entre le point de vue selon lequel les identités et les pratiques internationales ne peuvent changer, et le point de vue selon lequel tout est possible $»^{83}$. Pourtant, quand on regarde de plus près, comme nous l'avons vu plus tôt, cette " position moyenne » penche très nettement vers le positivisme. Selon Adler et Barnett, le constructivisme "devrait se distinguer clairement des approches poststructuralistes non scientifiques $»^{84}$, et les contributeurs à l'ouvrage de Katzenstein prétendent s'engager dans la « science normale, avec toutes ses exigences » quand ils "tentent d'en faire l'explication $»^{85}$. En outre, bien que Katzenstein affirme prendre ses distances par rapport au néoréalisme et au néolibéralisme, il se contente en fait d'élargir deux des postulats de base du néolibéralisme, en allant au-delà de sa conception d'un environnement international où les Etats sont perçus uniquement en termes de leurs capacités physiques, et en refusant de se limiter à la tendance des néolibéraux à s'intéresser exclusivement à l'impact des institutions sur les intérêts des Etats ${ }^{86}$.

La conception de la sécurité prônée par Katzenstein d'un côté, Adler et Barnett de l'autre, demeure assez conservatrice. Dans les deux cas, la sécurité concerne avant tout les Etats, comme l'indique clairement le titre de l'ouvrage dirigé par Katzenstein. De prime abord, Adler et Barnett semblent un peu plus aventureux en voulant ressusciter le concept de "communauté de sécurité », lancé par Karl Deutsch en 1957 et qui n'a jamais connu de lendemain ${ }^{87}$. Cependant, leur vision de la communauté, ou plutôt d'une " communauté de sécurité pluraliste », signifie simplement "une région transnationale composée de plusieurs Etats souverains $\aleph^{88}$. Adler et Barnett ont beau proclamer que leur approche "suggère non pas simplement une façon de repenser les questions de sécurité régionale, voire globale, mais plutôt un changement de paradigme dans la théorie des Relations Internationales ${ }^{89}$, leur vision de la sécurité reste assez traditionnelle. Ils sont préoccupés encore une fois par la recherche d'une voie moyenne, qui, cette fois-ci, offre "un mélange d'idéalisme - qui reconnaitrait les intérêts des Etats mais qui envisagerait la possibilité de progrès et la promesse d'institutions qui aideraient les Etats à surmonter leurs pires tendances - et le réalisme, dont les protagonistes principaux verraient le pire, mais continueraient à écrire sur les 
conditions qui pourraient fonder des changements pacifiques et de nouvelles formes d'organisation politique ${ }^{90}$. Autrement dit, la sécurité est avant tout une question de sécurité militaire, même si elle implique une communauté de normes et de valeurs.

Pour sa part, Katzenstein annonce d'emblée que lui et les autres contributeurs à son ouvrage adoptent "une définition étroite et traditionnelle des études de sécurité»" mais admet que «l'on perdrait beaucoup, et gagnerait peu, si des études de sécurité plus larges étaient comprimées dans le domaine bien développé et plus étroit des études stratégiques ${ }^{92}$. Donc, la forme de sécurité qui les intéresse avant tout est la sécurité militaire. Il reconnaît, cependant, les limites du constructivisme dominant en affirmant que l'évolution de la politique mondiale milite en faveur d'un « élargissement du domaine de la sécurité dans deux directions, pour comprendre des questions non militaires et des acteurs non étatiques" et accepte l'idée que les approches postpositivistes «offrent des défis plus profonds et plus dérangeants au domaine des études de sécurité nationale et à la théorie des Relations Internationales que les désaccords entre différents types de théorie explicative $»^{93}$.

Le concept de "communauté de sécurité " continue à être boudé par la plupart des constructivistes, malgré les espoirs d'Adler et Barnett, sans doute à cause des limites que lui impose la conjoncture internationale. Par contre, les idées de Katzenstein et son équipe ont soulevé beaucoup plus de controverses. Au cœur de leur analyse se trouve la notion d'identité, qui signifie avant tout l'identité nationale. Selon Katzenstein, l'identité des Etats «émerge de leurs interactions avec différents environnements sociaux, intérieurs et internationaux $\|^{94}$. L'identité est perçue comme une "étiquette comprenant diverses constructions de l'Etat et de la nation » et ce processus " est normalement politique et oppose des acteurs en conflit les uns avec les autres ${ }^{95}$. Les détails de ce processus ne l'intéressent pas particulièrement. Ce qui compte pour lui est le fait qu'un changement d'identité affecte les intérêts, ce qui influe sur la politique de sécurité nationale. C'est cette vision d'une relation linéaire entre identité et intérêts, qui reprend les idées de Wendt, que McSweeney rejette parce qu'elle ignore le fait que les intérêts et les identités s'affectent de façon réciproque ${ }^{96}$.

Enfin, et nous explorerons cette idée un peu plus loin, les constructivistes critiques se joignent à Jef Huysmans quand celui-ci reproche à Katzenstein de ne pas avoir abordé "la signification du langage dans les relations sociales", ce qui fait que "son cadre théorique se lit comme une sorte de sociologie institutionnelle qui ignorerait la signification du tournant dit linguistique dans la théorie sociale $»^{97}$. C'est un point central du constructivisme critique qui insiste sur la nécessité d'adopter une approche sociologique pour l'étude de la sécurité.

\section{Le constructivisme critique contre l'Ecole de Copenhague}

Si les constructivistes critiques prennent délibérément leurs distances avec les idées avancées par Katzenstein, en ignorant généralement celles d'Adler et Barnett, ils s'engagent directement dans un débat beaucoup plus élaboré avec les travaux issus de ce que l'on appelle l'Ecole de Copenhague ${ }^{98}$. L'intensité de l'intérêt que portent les constructivistes critiques à l'Ecole de Copenhague s'explique sans doute par le fait qu'elle existe depuis une quinzaine d'années, continue à publier, et constitue de loin le groupe universitaire qui a eu le plus d'impact sur le renouveau de la pensée sécuritaire depuis la fin de la Guerre Froide, du moins en Europe. Critiques et sympathisants reconnaissent tous la contribution de l'Ecole de Copenhague. Ainsi, Steve Smith 
présente ses travaux comme "l'un des développements les plus intéressants dans l'étude contemporaine de la sécurité $»^{99}$ et Bill McSweeney, malgré ses critiques, affirme que l'analyse méthodique des chercheurs de ce courant «a contribué de manière importante à notre compréhension du caractère relationnel [de la sécurité] et des insuffisances de sa définition étroite $»^{100}$.

Sur le plan de la théorie des Relations Internationales, l'approche de l'Ecole de Copenhague est presque inclassable. McSweeney considère la plupart de ses travaux comme « résolument objectivistes et réalistes ${ }^{101}$, tandis que Huysmans et Williams y voient un mélange de réalisme et de constructivisme ${ }^{102}$. Les ambiguïtés des positions théoriques de ce courant proviennent des points de départ de ses deux protagonistes principaux, Barry Buzan et Ole Wæver, et de leur évolution subséquente. Buzan s'est défini comme un néoréaliste structurel, dans la perspective de la pensée de Waltz, qui s'est peu à peu identifiée à l'Ecole anglaise et à son concept central de "société internationale». Wæver a insisté pour sa part sur sa double origine réaliste et postructuraliste, pour se qualifier de "réaliste postructuraliste» de «réaliste postsouverainiste ", et même de " constructiviste pessimiste " ${ }^{103}$.

En cherchant à renouveler la pensée de la sécurité, l'Ecole de Copenhague a introduit en particulier trois idées qui ont attiré l'attention des constructivistes critiques : celle d'une conception élargie de la sécurité, celle de la sécurité sociétale et celle de la sécurisation. Evidemment ces trois notions sont intimement liées, mais nous les séparerons ici pour les besoins de l'analyse.

$\mathrm{Au}$ cœur de la conception élargie de la sécurité de l'Ecole de Copenhague, on trouve quatre idées centrales. Premièrement, la sécurité doit être étudiée à la fois comme une pratique et un processus. Deuxièmement, la sécurité a des règles qui lui sont propres, et on ne doit donc pas confondre questions de sécurité et problèmes politiques. Troisièmement, les objets référents de la sécurité sont toujours des collectivités, et en premier lieu l'Etat. Enfin, quatrièmement, on peut appliquer la logique de la pratique de la sécurité à des domaines non strictement militaires. Pour qu'une question puisse représenter un enjeu de sécurité, il faut la «désignation d'une menace existentielle exigeant une action d'urgence ou des mesures spéciales et l'acceptation de cette désignation par un auditoire significatif »104. Donc la sécurité n'a pas de signification fixe et la logique d'une telle position permet de l'étendre au-delà du domaine militaire, pour y englober en particulier quatre autres secteurs, la politique, la société, l'environnement et l'économie. "La sécurité signifie la survie face à des menaces existentielles, mais ce qui constitue une menace existentielle varie selon le secteur considéré $»^{105}$.

Même si les chercheurs de l'Ecole de Copenhague proposent d'aller au-delà de la vision traditionnelle de la sécurité, les constructivistes critiques font preuve d'insatisfaction à l'égard de leurs idées sur plusieurs points. En premier lieu, ils attaquent Wæver, Buzan et al. pour leur stato-centrisme, qui n'est que peu atténué par l'idée d'une division entre sécurité nationale et sécurité sociétale. Tous rejettent la coupure entre intérieur et extérieur si importante dans les approches traditionnelles en Relations Internationales, et reprise par l'Ecole de Copenhague. Pour Bigo, "la délimitation classique entre sécurité intérieure et sécurité extérieure est perturbée ${ }^{106}$. Il refuse en fait totalement cette distinction. Il préfère parler en termes de champ de sécurité « où les différentes agences de sécurité (...) participent de facto à la redéfinition de leurs attributions respectives ", concept repris et élargi par Huysmans, qui le définit simplement comme 
" un champ de pratiques sécuritaires conceptualisé comme séparé, dans une grande mesure, des autres champs de pratiques ${ }^{107}$. Quant à McSweeney, qui tend à être une exception parmi les constructivistes critiques sur ce point, il opte pour une vision de la sécurité qui prend l'individu comme ultime objet référent ${ }^{108}$. Cela dit, il les rejoint en prônant une conception ouverte de la sécurité, qui rejette l'idée des cinq secteurs proposée par l'Ecole de Copenhague. Pour McSweeney, il faudrait une définition large de la sécurité qui tienne compte des besoins humains, qui comprenne les dimensions négatives et positives de la sécurité et qui mette l'accent sur la relation entre communautés, collectivités, Etats et individus comme source de sécurité ou d'insécurité109. Il adopte ainsi une vision assez proche des partisans des études critiques de sécurité. Pour leur part, Bigo et Huysmans préfèrent se limiter à indiquer les conditions qui entourent une définition de la sécurité. Pour Huysmans «une organisation spécifique d'énonciations en fait des énonciations de sécurité », tandis que Bigo s'intéresse beaucoup plus aux "pratiques de sécurisation/insécurisation qui traversent autant la sphère interne que la sphère externe $»^{110}$. Donc la sécurité est ce que l'on dit et ce que l'on fait, et il met en garde contre les «fausses continuités » qui feraient croire que la sécurité et l'insécurité seraient les mêmes à travers les ages ${ }^{111}$. Weldes et al. partagent largement cette analyse, et proposent la distinction entre construction et production des faits sociaux, dont la sécurité et les insécurités. La construction de la sécurité/insécurité concerne le discours, tandis que la production s'accomplit à travers les pratiques linguistiques et non linguistiques ${ }^{112}$. Ainsi, la plupart des constructivistes critiques ne veulent pas s'enfermer dans une "bonne » définition de la sécurité, d'où un intérêt particulier pour la pratique de la sécurité, et donc pour la notion de sécurisation, sur laquelle nous reviendrons ultérieurement.

Malgré sa tentative de distinguer cinq secteurs qui peuvent faire l'objet de mesures de sécurité, l'Ecole de Copenhague, et surtout Wæver, opte finalement pour une ontologie binaire, entre sécurité étatique ou nationale, qui touche la souveraineté, et la sécurité sociétale, qui concerne avant tout la question de l'identité ${ }^{113}$. La notion de sécurité sociétale est sans aucun doute celle qui a soulevé les critiques les plus acerbes contre les travaux de l'Ecole de Copenhague. Elle mérite d'être examinée de plus près et cela d'autant plus qu'elle a évolué depuis sa première formulation chez Buzan, qui voyait les menaces à la société surtout du point de vue de la sécurité de l'Etat ${ }^{114}$. Dans la première étude en profondeur du concept publiée par le groupe en 1993, Wæver en a proposé une définition assez statique :

«La sécurité sociétale concerne la capacité d'une société de persister dans son caractère essentiel sous des conditions changeantes et face à des menaces possibles ou réelles. Plus spécifiquement, il s'agit de la capacité de soutenir des modes traditionnels de langue, de culture, d'association, et d'identité religieuse et nationale et de coutumes dans des conditions acceptables pour leur évolution. (...) La sécurité sociétale concerne des situations où les sociétés perçoivent une menace en termes identitaires ${ }^{115}$.

Dans cette première version de la sécurité sociétale, les menaces principales provenaient des migrations et des identités en concurrence à l'intérieur ou à l'extérieur d'une société116. Deux ans plus tard, dans son célèbre article sur le concept de sécurisation, Wæver a cherché à dissiper tout malentendu qui ferait croire que la sécurité sociétale signifierait assurer la sécurité de l'Etat face aux sociétés qui constituent ce dernier, d'où son insistance sur la distinction entre sécurité nationale et sécurité sociétale à laquelle nous venons de faire allusion ${ }^{117}$. Il reconnaissait toutefois que le concept posait encore des problèmes, notamment celui de la voix de la société - 
qui parle en son nom et comment ? - et celui de considérer la société comme homogène, et sans différenciation. Comme nous le verrons, avec l'introduction du concept de sécurisation, Wæver a réussi à répondre, jusqu'à un certain point, à ceux qui l'accusaient de réifier la société et de donner à l'identité un caractère fixe et essentiel. Trois ans plus tard, cette conception beaucoup plus constructiviste de la sécurité sociétale est confirmée et renforcée. D'une part, toute confusion possible entre Etat et société est totalement rejetée, puisqu'on élargit la notion de société pour inclure presque toute collectivité, allant du village aux civilisations, en passant par les clans, les tribus, les nations, les religions et les races ${ }^{118}$. D'autre part, bien que l'on maintienne toujours l'identité comme l'objet référent central de la sécurité sociétale, les menaces à cette identité s'apparentent toujours à « la construction de quelque chose menaçant un 'nous' quelconque - et qui contribuent ainsi en fait à la construction ou à la reproduction de ce 'nous' $\aleph^{119}$. En outre, Wæver a raffiné sa conception des sources les plus courantes des menaces à la sécurité sociétale, en distinguant les migrations, la concurrence horizontale (menaces d'une culture voisine), la concurrence verticale (effets de projets intégrateurs sur le sens de l'identité), et éventuellement les effets de dépopulation ${ }^{120}$. Enfin, dans un texte publié en 2000 , Wæver tente de répondre à ceux qui craignent les conséquences normatives et politiques de ce concept, en mettant en garde contre la tentation de s'en servir pour construire une "politique de sécurité sociétale " et en exhortant les études dans ce domaine à s'intéresser plutôt aux " constellations identitaires et aux tentatives d'éviter dès le début le déclenchement d'inquiétudes au sujet de la sécurité sociétale $»^{121}$.

Tout en accueillant favorablement cette idée d'étendre la conception de la sécurité à la société, les constructivistes critiques manifestent un malaise évident vis-à-vis du concept de sécurité sociétale, tel qu'il est défini par Wæver, malgré l'évolution de sa pensée à ce sujet. Au fond de ce malaise se trouve ce que Huysmans appelle le "dilemme normatif d'écrire la sécurité » auquel font face tous les analystes. Chacun doit se poser la question : «Comment puis-je interpréter les problèmes de sécurité dans le domaine sociétal de façon à ce que je réduise le risque de reproduire la sécurisation même de ce domaine $»^{122}$ ? Pour être juste, il faut dire que Wæver lui-même est très conscient de ce problème, et prétend que « de telles questions éthiques devraient être au cœur d'un champ d'études de sécurité transformé en 'études de sécurisation' qui enquête sur qui peut sécuriser et sous quelles conditions ${ }^{123}$. Le moins que l'on puisse dire est que ce bémol de Wæver ne convainc pas ses critiques de la justesse de sa conception de la sécurité sociétale.

Les reproches les plus sévères à l'égard de ce concept viennent de McSweeney, qui rejette la conception de la société de l'Ecole de Copenhague comme "objectiviste et durkheimienne (...), qui a pour résultat une conception quasi-positiviste de l'identité », où on présente la société comme ayant une identité unique et qui n'indique aucun critère qui permettrait d'arbitrer entre les prétentions d'identités concurrentes. Ce faisant on oublie que « l'identité n'est pas un fait de société; elle est un processus de négociation entre les gens et les groupes d'intérêt». Enfin McSweeney reproche à Wæver et ses collègues de tenir pour acquis « sans preuve à l'appui » que la question de la survie d'une société est forcément seulement une question d'identité, et d'avoir ainsi une vision unidimensionnelle et partielle des menaces à la sécurité de la société ${ }^{124}$. Michael Williams vient à la rescousse de l'Ecole de Copenhague en affirmant que ceux qui accusent celle-ci de prétendre que la société a une seule identité passent à côté de la " radicalité » de sa " compréhension de la sécurité », car elle est très consciente du fait 
que le processus de sécurisation mène à la déclaration d'une "forme monolithique et réifiée de l'identité ${ }^{125}$. La lecture du concept de sécurité sociétale de l'Ecole de Copenhague proposée par Williams n'est manifestement pas partagée par les partisans du constructivisme critique.

Bigo se méfie aussi du concept de sécurité sociétale et l'utilisation que l'on risque d'en faire. Il note, en premier lieu, qu'aux Etats-Unis en particulier, la notion de sécurité sociétale est devenue synonyme de sécurité intérieure et de menaces non militaires ${ }^{126}$. Et de façon générale, il existe un vrai danger que l'on justifie "le principe d'une stratégisation de la sécurité intérieure, en donnant aux militaires l'argumentaire qui leur manquait », danger d'autant plus réel que les divers professionnels de la sécurité ne font pas de distinction entre sécurité interne et externe ${ }^{127}$. Mais Bigo garde ses critiques les plus sévères pour la décision de Wæver et ses collègues de désigner l'identité comme l'objet référent de la sécurité sociétale. Il fait remarquer que c'est sur cette question qu'internationalistes (c'est-à-dire, partisans de la distinction entre intérieur et extérieur) et sociologues (dans les faits, adeptes d'approches critiques) divergent. Les premiers « font de la sécurisation des identités une 'donnée' et justifient cette catégorie", tandis que les seconds "mettent l'accent sur l'origine sociale, les groupes qui l'énoncent, l'impossibilité d'avoir une représentation fixiste des identités ». Et Bigo s'inquiète des conséquences pratiques de la sécurisation des identités, qui ne concerne pas la survie, comme le prétendent les tenants de l'Ecole de Copenhague, mais "l'intolérance à l'égard des différences, [le] fait que le changement social et historique est perçu comme une menace $»^{128}$.

Derrière la conception de la sécurité de l'Ecole de Copenhague on retrouve le concept de sécurisation, sans aucun doute sa contribution la plus importante aux études de sécurité1 $^{129}$. Selon Wæver, qui est celui à qui on doit la formulation de cette idée, la sécurisation est le processus par lequel on produit la sécurité : "En nommant un certain développement un problème de sécurité, l'Etat peut réclamer un droit spécial, droit qui sera toujours défini, en dernière analyse, par l'Etat et ses élites $»^{130}$. La sécurisation signifie donc un processus où l'on nomme un objet référent de la sécurité et où l'on identifie les sources des menaces contre celui-ci. La première étape de ce processus est ce que Wæver appelle une " démarche sécurisante » (a securitizing move), un discours qui présente quelque chose comme une "menace existentielle». La tentative de sécurisation réussit dans la mesure où on arrive à convaincre son auditoire de la nécessité de cette démarche sécurisante ${ }^{131}$. En s'inspirant du philosophe anglais, J.L. Austin, Wæver conçoit la sécurisation comme un " acte de langage ", où :

« La sécurité elle-même ne présente aucun intérêt en tant que signe qui se réfère à quelque chose de plus réel ; l'énoncé lui-même est l'acte. En le disant, on accomplit quelque chose (...). En prononçant le mot 'sécurité', un représentant de l'Etat place un objet particulier dans un domaine spécifique, et ce faisant réclame un droit spécial d'utiliser tous les moyens nécessaires pour le bloquer ${ }^{132}$.

Toujours selon Wæver, pour réussir un acte de langage, il faut satisfaire trois conditions. Premièrement, il faut respecter l'exigence interne de l'acte de langage selon laquelle il faut suivre la "grammaire de la sécurité » et la "construction d'une trame comprenant une menace existentielle, un point de non-retour et une issue possible». Deuxièmement, l'acteur faisant la sécurisation doit tenir une position d'autorité. Enfin, la tentative de sécurisation réussira plus facilement si on se réfère à des objets qui sont généralement perçus comme menaçants ${ }^{133}$. 
Les constructivistes critiques n'éprouvent aucune difficulté à adopter ce concept, bien au contraire, puisqu'il met l'accent sur la construction sociale de la sécurité. Ils regrettent seulement que Wæver se soit arrêté à mi-chemin, que son concept demeure sous-développé. Leurs objections tournent autour de deux points principaux : Wæver propose une vision insuffisamment sociologique du processus de sécurisation ; sa théorisation de l'acte de langage ignore les aspects non linguistiques de la sécurisation.

Si Wæver déclare qu'« étudier la sécurisation c'est étudier les luttes de pouvoir autour d'un concept "134, comme nous l'avons vu, il ne pousse pas très loin cette idée, et se contente d'affirmer que c'est l'affaire des élites. C'est justement ce que lui reproche Bigo, qui rappelle que «la labellisation est toujours le produit d'un rapport de forces pour l'énoncé légitime $»^{135}$. De son côté, Huysmans, faisant écho à la critique de McSweeney, reproche à Wæver le fait qu'il n'indique jamais qui parle pour la société, car ce ne sont pas nécessairement les mêmes personnes que celles qui parlent au nom de l'Etat ${ }^{136}$. Bigo, fidèle à son refus d'établir une démarcation entre l'intérieur et l'extérieur, insiste sur l'importance du rôle joué par les professionnels de la sécurité (policiers, militaires, agences de renseignements, etc.) dans la formulation de la sécurité137, ce qui amène Wæver à répliquer que c'est la pratique et non pas les acteurs de la sécurisation qui doit être au centre de l'analyse ${ }^{138}$.

La deuxième série d'objections touche la thèse de la sécurisation comme acte de langage, si centrale dans le concept de Wæver. Elle rappelle aussi un des éléments-clés $\mathrm{du}$ constructivisme critique, l'importance du rôle socio-politique du langage. Cependant, sur la question du rôle du langage, on trouve des différences sensibles chez les constructivistes critiques, entre ceux pour qui le langage occupe une place primordiale dans la définition de la sécurité, et ceux qui y voient un aspect important, mais préconisent une approche plus sociologique. Bigo représente bien la position de ces derniers quand il affirme que la version de la sécurisation que présente Wæver est «trop linguistique et pas assez sociologique » et qu'elle ne distingue pas suffisamment «monde vécu et monde perçu » ${ }^{139}$. Tout en reconnaissant l'importance du discours, il souligne :

«La sécurisation n'est donc pas que de l'ordre des pratiques discursives, même si elle s'y origine. Elle est de l'ordre des pratiques non discursives, des technologies à l'œuvre, des effets de pouvoir, des luttes et plus particulièrement des compétitions institutionnelles au sein du champ de la sécurité $»^{140}$.

Pour leur part, Weldes et al. affirment aussi l'importance des pratiques non linguistiques, mais insistent sur le fait que les discours sont des sites de pouvoir, et cela d'au moins deux façons importantes. En premier lieu, certains discours sont plus puissants que d'autres parce qu'ils font partie du pouvoir institutionnel. Ensuite, parce que les discours portent la capacité de définir et de constituer le monde, "ces représentations d'insécurité sont elles-mêmes des sources importantes de pouvoir » ${ }^{141}$. Bien qu'ils soulignent l'importance du rôle joué par les représentants officiels de l'Etat dans ce processus, ils reconnaissent volontiers que d'autres groupes ou individus peuvent définir aussi des insécurités.

A l'autre extrême, on trouve Karin Fierke, qui s'inspire des travaux du philosophe Ludwig Wittgenstein ${ }^{142}$. Celui-ci concevait le langage comme un jeu ayant ses propres règles, et qui donnait une certaine signification, une structure, à une situation. Ainsi, selon Fierke, «le langage participe à la constitution du monde. L'utilisation du langage est une forme d'action en soi, qui est liée à d'autres types de pratiques $»^{143}$. Donc, elle ne 
traite pas directement de la question de la sécurisation en soi, et, comme cette citation l'indique, elle n'exclut pas nécessairement la possibilité d'une analyse beaucoup plus sociologique. On constate, cependant, que l'approche linguistique prônée par Fierke se concentre exclusivement sur les représentations du monde des décideurs, et ne nous dit rien au sujet des luttes politiques et sociales qui les ont précédées et qui ont contribué à leur formulation.

Enfin, Huysmans nous propose une approche qui combine les préoccupations linguistiques avec les besoins d'une sociologie de la sécurisation qui fait défaut chez Wæver. Comme ce dernier, Huysmans voit un énoncé de sécurité comme un acte de langage qui définit la sécurité. Il est donc performatif. Huysmans propose une « compréhension générique » du langage qui considère ces énoncés du point de vue de leur production, et qui comprend trois éléments : des pratiques d'énonciation de la sécurité, l'articulation d'un champ de sécurité et une " constellation de règles, ou une logique, qui organise des pratiques comme des pratiques de sécurité ", et qu'il appelle une " formation de sécurité ${ }^{144}$. Ainsi on arrive à définir la spécificité des pratiques de sécurité. Huysmans reconnaît qu'à travers sa théorie de l'acte de langage, Wæver propose une interprétation performative et générique du langage, mais qui reste dans un cadre qualifié de "réaliste néoclassique ", et qui tient pour acquis "un processus relativement bien institutionnalisé de formulation de politiques publiques à l'intérieur de l'Etat ». Selon Huysmans, Wæver ne théorise pas suffisamment le processus politique de la sécurisation, parce qu'il ne théorise pas la «culture politique et l'institutionnalisation des pratiques de sécurité au niveau de la société internationale $»^{145}$. Huysmans présente un programme de recherche en études stratégiques qui « théorise la mobilisation de la formation de sécurité d'un point de vue sociologique» et dont l'objet principal serait «l'institutionnalisation des environnements menaçants ${ }^{146}$. Cette approche se fonde sur le postulat selon lequel les énoncés de sécurité possèdent une capacité transformatrice et examine comment cette capacité est déterminée dans un contexte sociétal donné ${ }^{147}$.

La critique de la vision assez étriquée du processus de sécurisation proposée par Wæver constitue un des points forts du constructivisme critique. Au moment où la sécurité occupe la première place de l'ordre du jour international, une analyse sociologique de la sécurisation s'impose de plus en plus. La réduire à un simple acte de langage ne nous dit rien sur la complexité du processus qui a mené à la décision de déclarer tel ou tel domaine objet référent de la sécurité ou pourquoi on l'accepte si facilement.

Cela fait déjà une quinzaine d'années que le constructivisme est entré dans le vocabulaire de la théorie des Relations Internationales, et peu à peu certaines lignes directrices se précisent. Après avoir créé l'impression (et l'espoir) qu'une vision beaucoup plus critique des Relations Internationales, capable de mobiliser les esprits après des années de débat assez stérile entre néoréalisme et néolibéralisme, était née, il a fallu, au fil des années se rendre à l'évidence. Malgré un certain renouveau sur le plan ontologique, plus enclin à ouvrir et à explorer la boîte noire de l'Etat qu'à repenser en profondeur la nature des Relations Internationales, le constructivisme a pris la forme dominante que nous avons analysée dans la première partie de cet article, surtout aux Etats-Unis. Sans doute le commentaire de Karin Fierke sur la version du constructivisme proposée par Emmanuel Adler pourrait s'appliquer à une grande partie du courant dominant, quand elle déclare qu'il «tente de distancer le constructivisme des connotations négatives attachées au poststructuralisme, pour améliorer sa légitimité devant la communauté plus large des chercheurs en Relations 
Internationales, surtout aux Etats-Unis ${ }^{148}$. Mais doit-on s'en étonner? Après tout c'est le parcours habituel de tout paradigme qui aspire à remplacer celui qui est en place, comme nous le rappelle si bien Thomas Kuhn. C'est encore plus vrai quand la nouveauté d'aujourd'hui ne s'éloigne déjà pas beaucoup de l'orthodoxie d'hier. On peut y voir aussi une leçon pour le constructivisme critique qui ne peut, par définition, aspirer à devenir le nouveau paradigme de demain, mais qui ne peut se permettre non plus d'être marginalisé, ou bien en étant ignoré par ceux qui prétendent qu'il n'a rien de « constructif » à offrir, ou bien en se contentant de quelques mentions paternalistes qui le félicitent pour ses idées " intéressantes » ou " originales ».

Ces remarques sont encore plus vraies pour les études de sécurité. En fin de compte, malgré ses promesses, le constructivisme dominant a donné des résultats assez maigres dans ce domaine, autant sur le plan théorique que sur le plan empirique. Certes, l'ouvrage de Katzenstein a connu une certaine gloire. Il est devenu un point de référence incontournable, et peut certainement se targuer d'être la source la plus citée quand on parle des études de sécurité constructivistes. Le livre d'Adler et de Barnett n'a pas connu le même sort heureux, sans doute à cause de sa problématique relativement limitée. La proposition récente d'un programme constructiviste de recherche en études de sécurité nous suggère quelques raisons qui expliquent cette incapacité de percer dans un monde intellectuel où le positivisme s'impose encore. L'idée qui prédomine dans ce projet est celle de l'accommodement nécessaire avec le réalisme, au point où l'auteur parle du « danger de collaborer avec des théoriciens critiques, parce que cela pourrait saper l'engagement $d u$ constructivisme traditionnel avec le réalisme ${ }^{149}$. Décidément le constructivisme critique dérange ceux pour qui un programme constructiviste doit être "positivement positiviste et normativement neutre $»^{150}$, et dont l'objectif est de "bâtir la connaissance du monde et de contribuer aux débats de la majorité en relations internationales " ${ }^{151}$, tandis qu'aux "mains des constructivistes critiques, la théorie sociale est une arme pour livrer la guerre contre l'inégalité et l'injustice dans la politique mondiale $»^{152}$.

Si on peut regretter une tendance assez prévisible du constructivisme dominant à chercher légitimité et respectabilité, les constructivistes critiques peuvent se réjouir du fait que cela laisse un espace où ils peuvent continuer leurs propres réflexions et occuper une place privilégiée dans les débats en théorie des Relations Internationales. $\mathrm{Au}$ lieu de chercher à occuper le milieu du terrain, le constructivisme critique a tout à gagner à se maintenir aux alentours de la frontière entre constructivisme dominant, Théorie Critique et postmodernisme, seul endroit où il peut pratiquer pleinement l'ouverture et le dialogue qui font partie du réflexivisme qui le caractérise.

Le constructivisme critique peut prétendre à occuper cette place pour une autre raison fondamentale. Parmi les courants alternatifs en études de sécurité, il est un des seuls à pouvoir le faire. Il est vrai que les ambiguïtés sur le plan théorique de l'Ecole de Copenhague lui permettent d'être de tous les débats entre ceux qui ne croient pas que le militaire constitue l'unique champ de sécurité. Mais si elle ne semble éprouver aucune difficulté à reconnaître les études critiques de sécurité, et même à déclarer que leur approche "complète la nôtre "153, on remarquera que l'Ecole de Copenhague a montré peu de désir d'engager directement le débat avec les postmodernistes. Quant au dialogue avec le féminisme, son existence est encore plus périphérique. En revanche, comme nous avons tenté de le démontrer ici, non seulement le constructivisme critique emprunte volontiers des concepts, voire certaines formes de conceptualisation, à des 
approches plus radicales, mais les tenants de ces dernières n'hésitent pas à engager le débat avec lui.

\section{NOTES}

1. Sur les débuts du "troisième débat ", voir Lapid Y., "The Third Debate: On the Prospects of International Theory in a Post-positivist Era», International Studies Quarterly, 33, 3, 1989, pp. 235-254. Pour une excellente analyse des enjeux de ce débat, voir Wæver O., «Figures of international thought : introducing persons instead of paradigms », in Neumann I.B. et Wæver O. (dir.), The Future of International Relations. Masters in the Making, Londres et New York, Routledge, 1997, pp. 2-37.

2. Voir Wæver O., op cit., pp. 18-20.

3. Voir notamment Katzenstein P.J., Keohane R.O. et Krasner S.D., «International Organization and the Study of World Politics ", in Katzenstein P.J., Keohane R.O et Krasner S.D. (dir.), Exploration and Contestation in the Study of World Politics, Cambridge, Mass., The MIT Press, 1999, p. 6; Smith S., "The Increasing Insecurity of Security Studies: Conceptualizing Security in the Last Twenty Years ", in Croft S., Terrif T. (dir.), Critical Reflections on Security Studies, Londres, Frank Cass Publishers, 2000, p. 76.

4. La notion d'épistémologie est utilisée dans cet article dans son sens anglo-saxon d'étude des fondements de la connaissance.

5. «Dans l'anarchie, la sécurité est le but le plus élevé. C'est seulement si la survie est assurée que les Etats peuvent poursuivre en sécurité d'autres objectifs tels que la tranquillité, le profit, et la puissance ", Waltz K.N., Theory of International Politics, New York, Random House, 1979, p. 126. Toutes les traductions sont de l'auteur.

6. Notons que l'institutionnalisme néolibéral ne s'est jamais beaucoup intéressé aux questions de sécurité.

7. Voir notamment Ullman R.H., "Redefining Security", International Security, 8, 1, 1983, pp. 129-153; Buzan B., People, States and Fear: An Agenda for International Security Studies in the PostCold War Era, Brighton, Harvester Wheatsheaf, 1991 (1983), 393 p.; Nye J. et Lynn-Jones S., "International Security Studies: A Report on a Conference on the State of the Field", International Security, 12, 4, 1988, pp. 5-27; Tuchman Matthews J., «Redefining Security », Foreign Affairs, 68, 2, Council on Foreign Relations, 1989, pp. 162-177.

8. Nous adoptons les majuscules pour distinguer la Théorie Critique, liée au néogramscisme et à l'École de Francfort, des autres théories dites critiques.

9. Mearsheimer J., «The False Promise of International Institutions », International Security, 19, 3, 1994/1995, pp. 37-47.

10. Sterling-Folker J., «Competing Paradigms or Birds of a Feather? Constructivism and Neoliberal Institutionalism Compared », International Studies Quarterly, 44, 1, 2000, p. 98.

11. John Ruggie parle d'un «constructivisme postmoderne». Voir Ruggie J.G, Constructing the World Polity. Essays on International Institutionalism, Londres et New York: Routledge, 1998, p. 35. Ronald Jepperson, Alexander Wendt et Peter Katzenstein font allusion à une "position constructiviste radicale " adoptée par la "théorie poststructurelle des relations internationales ", voir Jepperson R.L, Wendt A. et Katzenstein P.J., « Norms, Identity and Culture in National Security ", in Katzenstein P.J. (dir.) The Culture of National Security: Norms and Identity in World Politics, New 
York, Columbia University Press, 1996, p. 46. Enfin, un des protagonistes les plus en vue du constructivisme dominant, Alexander Wendt, accroît la confusion en divisant le constructivisme en trois courants principaux, moderniste, postmoderniste et féministe. Voir Wendt A., Social Theory of International Politics, Cambridge, Cambridge University Press, 1999, pp. 3-4.

12. C'est la traduction que nous donnons aux termes « conventional » et « mainstream » que l'on retrouve constamment dans la littérature de langue anglaise.

13. Hopf T., "The Promise of Constructivism in International Relations Theory ", International Security, 23, 1, 1998, p. 182

14. Ibid., p. 196.

15. Jervis R., "Realism in the Study of World Politics ", in Katzenstein, Keohane, et Krasner, op. cit., p. 356.

16. Checkel J.T., «The Constructivist Turn in International Relations Theory ", World Politics, 50, 2, 1998, p. 325.

17. Ruggie J., op. cit., p. 34 .

18. Rengger N.J., International Relations, Political Theory and the Problem of Order. Beyond International Relations Theory?, Londres et New York, Routledge, 2000, p. 80. Adjectifsouligné dans le texte.

19. Steve Smith propose une bonne définition du positivisme, que nous adoptons ici, et qu'il décrit comme une "position méthodologique qui dépend d'une épistémologie empiriste qui fonde notre connaissance du monde sur la justification par l'expérience (...) et accepte la méthodologie et l'ontologie dans la mesure où elles sont empiriquement justifiées ». Voir Smith S., "Positivism and beyond », in Smith S., Booth K. et Zalewski M., (dir.), International Theory : positivism and beyond, Cambridge, Cambridge University Press, 1996, p.17. En théorie des Relations Internationales, le "postpositivisme» ne signifie pas une progression chronologique du positivisme à un stade supérieur, mais plutôt le refus de toute approche positiviste dans les sciences sociales. Pour la distinction entre "rationalisme» et "réflectivisme", voir Keohane R.O., «International Institutions : Two Approaches », International Studies Quarterly, 32, 4, 1988, pp. 379-396.

20. Une approche fondationnaliste prétend qu'il existe des bases sur lesquelles il est possible de justifier la connaissance, tandis que l'antifondationnalisme prétend le contraire et nie toute possibilité d'élaborer une grande théorie qui expliquerait le monde et qui permettrait de démontrer par le fait même la supériorité d'une théorie par rapport à une autre.

21. Smith S., "New Approaches to International Theories ", in Bayliss J. et Smith S. (dir.), The Globalization of World Politics, 2e édition, New York, Oxford University Press, 2001, p. 226. Pour une discussion plus détaillée de ces distinctions, voir Hollis M. et Smith S., Explaining and Understanding International Relations, Oxford, Clarendon Press, 1990, pp. 45-91.

22. Onuf N., "Constructivism: A User's Manual », in Kubálková V., Onuf N.et Kowert P. (dir.), International Relations in a Constructed World, Armonk, New York, M. E. Sharpe, 1998, p. 59.

23. Wendt A., op. cit., p. 1.

24. Smith S., "Foreign Policy Theory and the New Europe ", in Carlsnaes W. et Smith S. (dir.), European Foreign Policy. The EC and Changing Perspectives in Europe, Londres, Sage Publications, 1994, p. 16.

25. Wendt A., op.cit. p. 1 .

26. Ibid., p. 11.

27. Voir Hopf T., op.cit., p. 182 ; Checkel J.T., op. cit., p. 327 ; Wendt A., op. cit., pp 39-40; et Kubálková V., Onuf N. et Kowert P., «Constructing Constructivism », in Kubálková V., Onuf N. et Kowert P., op. cit., pp. 19-20.

28. Katzenstein P.J., The Culture of National Security. Norms and Identity in World Politics, New York, Columbia University Press, 1996.

29. Wendt A., op. cit., p. 2.

30. Adler E., « Seizing the Middle Ground: Constructivism in World Politics », European Journal of International Relations, 3, 3, London, Sage Publications, 1997, pp. 319-363. 
31. Finnemore M. et Sikkink K., "International Norm Dynamics and Political Change », in Katzenstein P.J., Keohane R.O., et Krasner S.D, op. cit., p. 269.

32. McSweeney B., Security, Identity and Interests. A Sociology of International Relations, Cambridge, Cambridge University Press, 1999, p. 203.

33. Smith S., "New Approaches to International Theories", op.cit., p. 187. On peut assimiler « rationalistes » et « réflectivistes » aux positivistes et postpositivistes.

34. Katzenstein P.J., Keohane R.O. et Krasner S.D., op. cit., p. 42.

35. Wæver O., «Figures of international thought: introducing persons instead of paradigms", op. cit., p. 25.

36. Marcus G., « Foreward », in Weldes J., Laffey M., Gusterson H., et Duvall R. (dir.), Cultures of Insecurity: States, Communities, and the Production of Danger, Minneapolis et Londres, University of Minnesota Press, 1999, p. 10.

37. Fierke K.M., "Critical Methodology and Constructivism », in Fierke K.M. et Jørgensen K.E., Constructing International Relations: The Next Generation, Armonk, New York, M. E. Sharpe, 2001, pp. 115-135.

38. Weldes J., Laffey M., Gusterson H., et Duvall R., «Introduction: Constructing Insecurity », in Weldes, Laffey, Gusterson, et Duvall, op. cit., p. 13.

39. Sur la question du réflexivisme en théorie des Relations Internationales, voir Neufeld M., The Restructuring of International Relations, Cambridge, Cambridge University Press, 1999, pp. 39-69.

40. Ce que reconnaît volontiers David Campbell, avec un plaisir manifeste, dans son épilogue de Writing Security. United States Foreign Policy and the Politics of Identity, 2e édition, Minneapolis, University of Minnesota Press, 1998, pp. 222-225. Dans ces quelques pages, Campbell fait bien ressortir les différences entre le postmodernisme et le constructivisme critique ainsi que la possibilité de dialogue entre les deux.

41. Der Derian J., "The value of security: Hobbes, Marx, Nietsche, and Baudillard ", in Lipschutz

R. D., (dir.), On Security, New York, Columbia University Press, p. 27.

42. McSweeney, op. cit., p. 30.

43. Smith S., "The Increasing Insecurity of Security Studies: Conceptualizing Security in the Last Twenty Years ", op. cit., p. 88.

44. Booth K., "Security and Self: Reflections of a Fallen Realist », in Krause K. et Williams M.C, (dir.), Critical Security Studies: Concepts and Cases, Minneapolis, University of Minnesota Press, 1997, p. 108.

45. Parmi les textes néogramsciens en Relations Internationales, citons Cox R., "Social Forces, States and World Orders: Beyond International Relations Theory ", Millennium, 10, 1, 1981, pp. 126-155, et Gill S. (dir.), Gramsci, Historical Materialism and International Relations, Cambridge, Cambridge University Press, 1993, 320 p. L'apport de l'Ecole de Francfort apparaît en particulier dans Linklater A., Beyond Marxism and Realism: Critical Theory and International Relations, Basingstoke, Macmillan, 1990, 216 pages et Neufeld M., op. cit.Richard Wyn Jones propose même un troisième courant, inspiré des travaux de Jürgen Habermas, lui-même considéré comme un des héritiers de l'Ecole de Francfort, voir Jones R.W., «Introduction: Locating Critical International Relations Theory ", in Jones R.W.(dir.), Critical Theory and World Politics, Boulder, Lynne Rienner Publishers, 2001, p. 7.

46. Rengger, op. cit., p. 150.

47. Krause K. et Williams M.C., « Preface:Toward Critical Security Studies », in Krause et Williams, op. cit., pp. 10-11.

48. Krause K. et Williams M.C., "From Strategy to Security: Foundations of Critical Security Studies ", in Krause et Williams, op. cit., p. 50.

49. Ibid., p. 35.

50. Krause K. et Williams M.C., « Preface: Toward Critical Security Studies », op. cit., p. 14.

51. Ibid., p. 13. 
52. Booth K., op. cit., p. 110.

53. Wyn Jones R., Security, Strategy and Critical Theory, Boulder, Lynne Rienner Publishers, 1999, p. 103.

54. Ibid., p. 95.

55. Ibid., p. 115.

56. On remarquera, par exemple, que la maison d'édition Lynne Rienner, spécialiste bien connue des études en Relations Internationales aux Etats-Unis, propose une série spéciale appelée "Critical Security Studies », mais n'y a publié en fait qu'un seul ouvrage, celui de Wyn Jones, et se prépare, quelques cinq ans plus tard, à en publier un deuxième en octobre 2004, sous la direction de Ken Booth, intitulé Critical Security Studies and World Politics.

57. Katzenstein P.J., Keohane et Krasner, op. cit., p. 38.

58. Pour une bonne présentation de l'apport du postmodernisme à la théorie des Relations Internationales, voir Devetak R., "Postmodernism » in Burchill S. et al., Theories of International Relations, 2e édition, Londres, Palgrave, 2001, pp. 181-208.

59. Vasquez J.A., "The Post-Positivist Debate: Reconstructing Scientific Enquiry and International Relations Theory After Enlightenment's Fall », in Booth K. et Smith S. (dir.), International Relations Theory Today, Cambridge, Polity Press, 1995, p. 218.

60. Rosenau P.M., Post-modernism and the Social Sciences. Insights, Inroads, and Intrusions, Princeton et Chichester, Princeton University Press, 1992, p. 3.

61. Ibid., p. 6. Selon un dictionnaire de la pensée postmoderne : «Le poststructuralisme est un terme générique utilisé pour désigner toutes les théories rejetant les principes du structuralisme, qui, des années 1950 aux années 1970, constituait le paradigme principal du questionnement intellectuel en France ", Sim S. (dir.), The Icon Critical Dictionary of Postmodern Thought, Cambridge, Icon Books, 1998, p. 341.

62. S'ils rejettent totalement le néoréalisme, les postmodernistes adoptent une position plus nuancée à l'égard du réalisme classique. Tout en critiquant la " pauvreté du néoréalisme ", Richard Ashley reconnaît que le réalisme est "beaucoup plus fidèle à la pratique de la politique mondiale ", Ashley R.K., "The Poverty of Neorealism ", International Organization, 38, 2, 1984, p. 275. Selon Bradley Klein on doit voir le réalisme comme «l'une des représentations possibles de l'espace global et des pratiques globales ", Klein B.S., Strategic Studies and World Order; The Global Politics of Deterrence, Londres, CSIR, Cambridge University Press, 1994, p. 35. Walker R. J. B. le voit comme «le site d'un vaste nombre d'affirmations contestées et de disputes métaphysiques", Inside/outside: international relations as political theory, Cambridge, Cambridge University Press, p. 105.

63. Klein B., op. cit., p. 12.

64. Walker R.J.B., « The Subject of Security », in Krause K. , Williams M.C., op. cit., pp. 69-70.

65. Campbell D., op. cit., p. 71.

66. Ibid., p. 68.

67. Klein B., op. cit., p. 36.

68. Walker R.J.B., « The Subject of Security », op. cit., p. 69.

69. Der Derian J., op. cit., p. 26.

70. Campbell D., op. cit., pp. 202-203.

71. Walker R.J.B., « The Subject of Security », op. cit., pp. 70-71.

72. George J., Discourses of Global Politics: A Critical (Re)Introduction to International Relations, Boulder, Lynne Rienner Publications, 1994, p. 211.

73. Klein B., op. cit., p. 139.

74. Dalby S., «Contesting an Essential Concept: Reading the Dilemmas in Contemporary Security Discourse », in Krause K. et Williams M.C., op. cit., pp. 12-13.

75. Walker R.J.B., Inside/outside, op. cit., p. 139.

76. Campbell D., op. cit., p. 171.

77. Doty R.L., «Immigration and the Politics of Immigration », in Cafetz G., Spirtas M. et Frankel

B. (dir.), The Origins of National Interests, Londres et Portland, Frank Cass, 1999, pp. 72-73. 
78. Ibid., pp. 77-80.

79. Adler E., Barnett M. (dir.), Security Communities, Cambridge, Cambridge University Press, 1998. 80. Adler E. et Barnett M., "Security communities in theoretical perspective ", in Adler E. et Barnett M., op. cit., p. 10.

81. McSweeney B., op. cit., p. 30.

82. Katzenstein P.J., "Conclusion: National Security in A Changing World », in Katzenstein P.J., The Culture of National Security, op. cit., p. 528.

83. Adler E. et Barnett M., "Security communities in theoretical perspective », op. cit., p. 15

84. Ibid., p. 12.

85. Jepperson R.L., Wendt A. et Katzenstein P.J., op. cit., p. 65.

86. Katzenstein P.J., "Introduction: Alternative Perspectives on National Security", in Katzenstein P.J., op. cit., pp. 16-17.

87. Deutsch K. et al., Political Community and the North Atlantic Area, Princeton, Princeton University Press, 1968, $227 \mathrm{p}$.

88. Adler E. et Barnett M., " A framework for the study of security communities ", in Adler E. et Barnett M., op. cit., p. 30.

89. Ibid., p. 59.

90. Adler E., Barnett M., « Security communities in theoretical perspective », op. cit., pp. 14-15.

91. Katzenstein P.J., « Introduction: Alternative Perspectives on National Security », op. cit., p. 10.

92. Ibid., p. 9, note 23 .

93. Katzenstein P.J., "Conclusion: National Security in A Changing World », in Katzenstein P.J., op. cit., p.p. 524-525.

94. Katzenstein P.J., « Introduction: Alternative Perspectives on National Security », op. cit., p. 24.

95. Ibid., p. 6.

96. McSweeney P., op. cit., p. 130.

97. Huysmans J., « Defining Social Constructivism in Security Studies: The Normative Dilemma of Writing Security ", Alternatives, 27, supplément, 2002, p. 44.

98. C'est Bill McSweeney qui a popularisé cette étiquette en 1996 dans un article intitulé "Identity and Security : Buzan and the Copenhagen School ", Review of International Studies, 22, 1, 1996, 81-93, en faisant référence au groupe de recherche du Centre for Peace and Conflict Research de l'Université de Copenhague, dirigé par Ole Wæver et Barry Buzan. En 1990 ils ont publié leur premier ouvrage collectif : Buzan B., Kelstrup M., Lemaître P., Tromer E. et Wæver O., The European Security Order Recast. Scenarios for the Post-Cold War Era, Londres, Pinter Publishers, 282 p. Les travaux les plus cités du groupe sont: Wæver O., Buzan B., Kelstrup M. et Lemaître P., Identity, Migration and the New Security Agenda in Europe, New York: St. Martin's Press, 1993, 221 p.; Wæver O., "Securitization and Desecuritization », in. Lipschutz R.D (dir.), On Security, op. cit., pp. 46-86 et Buzan B., Wæver O. et de Wilde J., Security: A New Framework for Analysis, London and Boulder, Colorado, Lynne Rienner Publishers, 1998, 239 p. Leur dernier ouvrage est Buzan B. et Wæver O., Regions and Powers: The Structure of International Society, Cambridge, Cambridge University Press, 2004, 564 p. Pour une synthèse des idées de ce courant en français, voir Wæver O., «Insécurité, identité : une dialectique sans fin », in Le Gloannec A.M., Entre union et nations. L'Etat en Europe, Paris, Presses de Sciences-Po, 1998, pp. 91-138.

99. Smith S., « The Increasing Insecurity of Security Studies: Conceptualizing Security in the Last Twenty Years ", op. cit., p. 86.

100. McSweeney B., Security, Identity and Interests, op. cit., p. 78.

101. Ibid., p. 69.

102. Voir Huysmans J., op. cit., p. 54 et Williams M.C, «Words, Images, Enemies: Securitization and International Politics », International Studies Quarterly, 47, 4, 2003, p. 512.

103. Pour le néoréalisme de Buzan, voir People, States and Fear, op. cit., et Buzan B., Jones C.A. et Little R., The Logic of Anarchy: Neorealism to Structural Realism, New York, Columbia University 
Press, 1993, 267 p. Les diverses positions de Wæver sont expliquées dans « Security Analysis: Conceptual Apparatus ", in Buzan B., Wæver et de Wilde, op. cit., p. 47 note 7, et Wæver O., "The EU as a security actor: Reflections from a pessimistic constructivist on post-sovereign security actors ", in Kelstrup M. et Williams M.C. (dir.), International Relations and the Politics of European Integration: Power, Security and Community, Londres et New York, Routledge, 2000, p. 287, note 17. Dans un article publié en 1997, Buzan et Wæver ont défini leur conception de la sécurité comme «constructiviste jusqu'au bout », Buzan B. et Wæver O., "Slippery? Contradictory? Sociologically Untenable?: The Copenhagen School Replies », Review of International Studies, 23, 2, 1997, p. 245.

104. Buzan B., Wæver O. et de Wilde J., op. cit., p. 27.

105. Ibid.

106. Bigo D., «L’Europe de la sécurité intérieure : penser autrement la sécurité », in Anne-Marie Le Gloannec, op. cit., p. 56.

107. Bigo D., "When two become one: Internal and external securitisations in Europe ", in Kelstrup et Williams, op. cit., p. 174; Huysmans, op. cit., p. 45.

108. McSweeney B., op. cit., p. 99.

109. Voir ibid., pp. 99-100.

110. Huysmans J., op.cit., p. 45 et Bigo D., "When two become one: Internal and external securitisations in Europe ", op. cit., pp. 177-178.

111. Voir Bigo D., Polices en réseaux : l'expérience européenne, Paris, Presses de Sciences-Po, 1996, pp. 252-253.

112. Weldes J., Laffey M., Gusterson H., et Duvall R., op. cit., pp. 16-17.

113. Wæver O., "Societal Security: The Concept ", in Wæver O., Buzan B., Kelstrup M. et Lemaître P., op. cit., p. 25 ; et Wæver O., « Securitization and Desecuritization », op. cit., p. 67.

114. Voir Buzan B., People, States and Fear, op. cit., pp. 122-123.

115. Wæver O., «Societal Security: The Concept », op. cit., p. 23. Wæver reprend presque mot pour mot la définition originelle du terme proposée deux ans plus tôt par Buzan dans People, States and Fear, op. cit., p. 123.

116. Voir Wæver O., « Societal Security: The Concept », op. cit., pp. 42-46.

117. Sur l'évolution de la réflexion sur le concept de sécurité sociétale, voir Wæver 0 ., "Securitization and Desecuritization ", op. cit., pp. 67-71.

118. Buzan B., Wæver O. et de Wilde J., op. cit., p. 123.

119. Ibid., p. 120. Mot souligné dans le texte.

120. Ibid., p. 121.

121. Wæver O., « The EU as a security actor », op. cit., pp. 253-254.

122. Huysmans J., op. cit., p. 47.

123. Wæver O., " The EU as a security actor ", op. cit., p. 252.

124. McSweeney B., op. cit., pp. 69-78. Pour une critique semblable, voir Theiler T., «Societal security and social psychology ", Review of International Studies, 29, 2, 2003, pp. 250-258.

125. Williams M.C., op. cit., p. 519.

126. Bigo D., «When two become one: Internal and external securitisations in Europe », op. cit., p. 191

127. Bigo D., « L’Europe de la sécurité intérieure : penser autrement la sécurité », op. cit., pp. 71-72.

128. Ibid., pp. 83-84.

129. Pour une analyse en profondeur de ce concept, voir Williams M.C., "Words, Images, Enemies : Securitzation and International Politics », op. cit., et Ceyhan A., « Analyser la sécurité : Dillon, Wæver, Williams et les autres », Cultures \& Conflits, 31-32, 1998, pp. 49-57.

130. Wæver O., "Securitization and Desecuritization », op. cit., p. 54. Mots soulignés dans le texte.

131. Voir Buzan B., Wæver O. et de Wilde J., op. cit., pp. 25-26.

132. Wæver O., «Securitization and Desecuritization », op. cit., p. 55. Mot souligné dans le texte. 
133. Voir Wæver O., "The EU as a Security Actor », op. cit., pp. 252-253, et Buzan B., Wæver O. et de Wilde J., op. cit., pp. 32-33.

134. Buzan B., Wæver O. et de Wilde J., op. cit., p. 32.

135. Bigo D., «L'Europe de la sécurité intérieure : penser autrement la sécurité », op. cit., p. 69.

136. Huysmans J., op. cit., p. 55.

137. Sur ce sujet, voir notamment son livre Polices en réseaux, op. cit.

138. Buzan B., Wæver O. et de Wilde J ; op. cit., pp. 32-33.

139. Bigo D., « L’Europe de la sécurité intérieure : penser autrement la sécurité », op. cit., pp. 69-70.

140. Bigo D., « Sécurité et immigration : vers une gouvernementalité par l'inquiétude ? ", Cultures \& Conflits, 31-32, 1998, p. 27.

141. Weldes J., Laffey M., Gusterson H., et Duvall R., op.cit., pp. 17-18. Weldes analyse plus longuement l'importance des pratiques non linguistiques dans la construction des intérêts, dont la sécurité, dans Weldes J., Constructing National Interests: The United States and the Cuban Missile Crisis, Minneapolis et Londres, University of Minnesota Press, 1999, pp. 109-112.

142. Voir par exemple, Fierke K.M., "Changing World of Security ", in Krause K. et Williams M.C., op. cit., pp. 233-252 ; Fierke K.M., «Critical Methodology and Constructivism », op. cit. ; et Fierke K.M.,Wiener A., "Constructing Institutional Interests: EU and NATO Enlargement», in Christiansen T., Jørgensen K.E. et Wiener A. (dir.), The Social Construction of Europe, Londres, Sage Publications, 2001, pp. 121-139.

143. Fierke K.M., « Critical Methodology and Constructivism », op. cit., p. 126.

144. Voir Huysmans J., op. cit., pp. 45-46.

145. Ibid., p. 55.

146. Ibid., p. 53.

147. Ibid., p. 57.

148. Fierke K.M., «Critical Methodology and Constructivism », op. cit., p. 121.

149. Farrel T., "Constructivist Security Studies: Portrait of a Research Program », International Studies Review, 4, 1, 2002, p. 59.

150. Ibid., p. 62.

151. Ibid., p. 72. Il n'est donc pas étonnant d'entendre parler aujourd'hui de "constructivisme réaliste ». Voir Barkin J.S., "Realist Constructivism », International Studies Review, 5, 3, 2003, pp. 325-342.

152. Ibid., p. 59.

153. Buzan B., Wæver O. et de Wilde J., op. cit., p. 35.

\section{RÉSUMÉS}

L'arrivée du constructivisme vers la fin des années 1980 semblait apporter une véritable approche de rechange aux théories néoréalistes et néolibérales qui dominaient le débat en Relations Internationales. Cependant, après les premiers élans d'enthousiasme il fallut se rendre à l'évidence que, malgré ses promesses, le constructivisme dominant qui était en train de s'imposer, surtout en Amérique du Nord, constituait un courant très large, qui pouvait s'accommoder facilement avec les approches positivistes. Dans le domaine des études de sécurité, ce constructivisme dominant a produit quelques travaux intéressants, mais qui reflètent une prudence profondément ancrée. Il n'est donc pas étonnant de voir émerger une tendance 
constructiviste critique, beaucoup plus postposiviste, et qui ne refuse pas le dialogue avec le postmodernisme et la Théorie Critique. Cette approche ne se confond pas avec les «études critiques de la sécurité » et se distingue en particulier par ses appréciations critiques des travaux de l'école de Copenhague.

The arrival of constructivism towards the end of the 1980s appeared to herald a truly alternative approach to the neorealist and neoliberal theories which dominated the debate in International Relations. However, after the first surge of enthusiasm, it became clear that, despite its promises, the mainstream constructivism which was beginning to take hold, especially in North America, was a very broad current that could easily fit in with positivist approaches. In the field of security studies, this mainstream constructivism has produced some interesting work, but which reflects a deeply ingrained cautiousness. So there is no surprise to see a critical constructivist trend emerge, which is much more postpositivist, and which does not refuse to dialogue with postmodernism and Critical Theory. This approach is not to be confused with "critical security studies" and is dintinguished in particular by its critical assessment of the work done by the Copenhagen School.

INDEX

Mots-clés : Relations Internationales, constructivisme, études critiques, sécurité

\section{AUTEUR}

\section{ALEX MACLEOD}

Alex Macleod est professeur au département de science politique de l'Université du Québec à Montréal et directeur du Centre d'études des politiques étrangères et de sécurité de l'UQAM. 